\title{
Formation of an Intricate Helical Bundle Dictates the Assembly of the 26S Proteasome Lid
}

\author{
Eric Estrin, ${ }^{1}$ José Ramón Lopez-Blanco, ${ }^{3}$ Pablo Chacón, ${ }^{3}$ and Andreas Martin ${ }^{1,2, *}$ \\ ${ }^{1}$ Department of Molecular and Cell Biology \\ ${ }^{2}$ QB3 Institute \\ University of California, Berkeley, Berkeley, CA 94720, USA \\ ${ }^{3}$ Department of Biological Physical Chemistry, Rocasolano Physical Chemistry Institute, CSIC, Serrano 119, 28006 Madrid, Spain \\ ${ }^{*}$ Correspondence: a.martin@berkeley.edu \\ http://dx.doi.org/10.1016/j.str.2013.06.023
}

\section{SUMMARY}

The $26 \mathrm{~S}$ proteasome is the major ATP-dependent protease in eukaryotes and thus involved in regulating a diverse array of vital cellular processes. Three subcomplexes form this massive degradation machine: the lid, the base, and the core. While assembly of base and core has been well-studied, the detailed molecular mechanisms involved in formation of the nine-subunit lid remain largely unknown. Here, we reveal that helices found at the $C$ terminus of each lid subunit form a helical bundle that directs the ordered self-assembly of the lid subcomplex. Furthermore, we use an integrative modeling approach to gain critical insights into the bundle topology and provide an important structural framework for our biochemical data. We show that the helical bundle serves as a hub through which the last-added subunit Rpn12 monitors proper lid assembly before incorporation into the proteasome. Finally, we predict that the assembly of the COP9 signalosome depends on a similar helical bundle.

\section{INTRODUCTION}

The $26 \mathrm{~S}$ proteasome is a massive, $\sim 2.5 \mathrm{MDa}$ molecular machine that is responsible for targeted protein degradation in eukaryotes, and, thus, has vital roles in regulating diverse cellular processes such as the cell cycle, transcription, and protein quality control (Glickman and Ciechanover, 2002). Proteins destined for degradation are tagged with an ubiquitin chain that targets them to the proteasome for ATP-dependent unfolding and hydrolysis of the polypeptide (Thrower et al., 2000).

The proteasome can be separated into the core particle and the regulatory particle, which can be further subdivided into the base and lid subcomplexes. The core particle is composed of two copies of 14 different subunits ( $\alpha 1-7$ and $\beta 1-7)$, forming a barrel-shaped structure whose internal chamber houses the peptidase active sites. The base sits atop the core and is responsible for ubiquitin recognition, substrate unfolding, and translocation of the unfolded polypeptide into the core particle, while the lid binds to the side of the base and core particle and deubi- quinates substrates prior to degradation. The base is composed of a heterohexameric ring of ATPases (Rpts 1-6), an ubiquitin receptor (Rpn13), and two large non-ATPase subunits (Rpn1 and Rpn2). Another intrinsic ubiquitin receptor (Rpn10) associates with the base and lid upon their assembly into the regulatory particle (Glickman et al., 1998). The lid is composed of six $\mathrm{PCl}-d o m a i n-c o n t a i n i n g ~ p r o t e i n s ~(R p n 3 / 5 / 6 / 7 / 9 / 12)$, two MPNdomain-containing proteins (Rpn8/11), and one peptide, Sem1. The only catalytically active member of the lid is Rpn11, which serves as the essential deubiquitinase of the proteasome. The $\mathrm{PCl}$ domains form a horseshoe shape with finger-like extensions that radiate out, contacting subunits in the core particle and the $\mathrm{AAA}$ + ring of the base, while the MPN domains heterodimerize and predominantly interact with Rpn2 (Lander et al., 2012; Pathare et al., 2012). Notably, both the COP9 signalosome (CSN) and eukaryotic initiation factor 3 (elF3) harbor six $\mathrm{PCl}-$ domain-containing subunits as well as two MPN-domaincontaining subunits and are thought to be distantly related to the lid (Scheel and Hofmann, 2005).

The $26 \mathrm{~S}$ proteasome is essential in all eukaryotic cells, and the assembly of this large intricate complex poses a number of challenges. Large protein complexes often contain hetero-oligomeric assemblies of homologous subunits that in many cases are thought to have evolved by duplication and diversification from an ancient precursor within a homo-oligomer (PereiraLeal et al., 2007). The proteasome is composed of a number of subassemblies that each harbor highly homologous subunits. For instance, the seven $\alpha$ and seven $\beta$ subunits, which form the outer and inner rings of the core peptidase, exhibit high homology (Hughes, 1997). Similarly, the AAA+ unfoldase in the base is composed of a heterohexameric assembly of the highly homologous ATPase subunits Rpt1-6, and the lid structure is dominated by an assembly of six distinct, but related, $\mathrm{PCl}$ domains (Serino and Pick, 2013). Although this diversification may allow for the specialization of individual subunits, it also might lead to more complicated assembly processes and a tendency of subunits to occupy the wrong position within a complex due to strong homology with other constituents. Subunit misassembly could lead to inactive complexes, or worse, to complexes that poison a cell. This burden may be especially high considering that one misplaced subunit has the potential of turning an otherwise useful assembly of numerous proteins into an inactive complex.

To prevent these assembly issues, large protein complexes therefore use a number of design principles to ensure their 
accurate and efficient maturation. In some cases, complex-specific assembly factors favor the association between appropriate partners. For example, proteasome-specific chaperones ensure the proper arrangement of the $\alpha$ subunits within the core particle (Kusmierczyk et al., 2008) and the correct order of Rpt subunits in the heterohexameric $\mathrm{AAA}+$ ring of the base (Funakoshi et al., 2009).

In addition to this strategy for correct subunit arrangement, many complexes rely on mechanisms that inhibit premature catalytic activity of assembly intermediates to prevent futile substrate processing. The proteasome utilizes such mechanisms to suppress its proteolytic activity until full maturation. The three proteolytically active $\beta$ subunits of the core particle are inhibited by $\mathrm{N}$-terminal propeptides until the complete assembly of the core particle triggers the autocatalytic removal of these sequences. This ensures that proteolysis is only activated once the catalytic sites are sequestered in the core particle internal chamber (Arendt and Hochstrasser, 1999; Chen and Hochstrasser, 1996). Another strategy is to strictly control substrate access to these active sites. In the proteasome core particle, the $\mathrm{N}$ termini of the $\alpha$ subunits form a gate that excludes folded proteins and large unfolded polypeptides from the proteolytic chamber (Groll et al., 2000). This gate is opened only upon binding of a properly assembled base that recognizes appropriate substrates and actively translocates them into the core (Smith et al., 2007). Moreover, the deubiquitinase activity of Rpn11 is inhibited until the lid is incorporated into the regulatory particle (Verma et al., 2002). Although there are hypotheses on how this regulation of Rpn11 may occur, the mechanistic basis for this observation is unknown (Beck et al., 2012; Lander et al., 2012).

Large complexes are often assembled in parts, with individual subunits initially forming smaller subcomplexes that are combined into the holoenzyme only upon their proper completion (Sauer and Baker, 2011; Staley and Woolford, 2009). This may be advantageous because smaller pieces likely assemble more easily and controlled more than one massive construction. Such modularity may also allow the quick regulation of complex levels, the ability to use interchangeable, functionally distinct parts, and the easier transport between organelles. Based on current models, the $26 \mathrm{~S}$ proteasome also appears to assemble from preformed subcomplexes (Murata et al., 2009; Tomko and Hochstrasser, 2013), which, for instance, allows their separate import into the nucleus (Isono et al., 2007).

Although the assembly factors and the biogenesis for the base and the core particle have been well-studied, the detailed mechanisms involved in lid assembly remain largely unknown. We have recently shown that Escherichia coli-expressed lid resembles the endogenous yeast lid, both in structure and function, suggesting that there are no essential factors dedicated to lid assembly (Lander et al., 2012). Previous cryo-electron microscopy (EM) reconstructions revealed that the six $\mathrm{PCl}$ domains of the lid are arranged in a horseshoe-shaped structure that appears to act as a scaffold and stabilize the lid through substantial lateral interactions between $\mathrm{PCl}$-containing subunits (da Fonseca et al., 2012; Lander et al., 2012; Lasker et al., 2012). Additionally, a number of subassemblies of the lid have been detected in vivo and upon subcomplex dissociation in vitro, and these observations have given rise to an assembly model wherein Rpn5/8/9/11 associate first, followed by the ordered additions of Rpn6, Rpn3/7, and finally Rpn12 (Fukunaga et al., 2010; Sharon et al., 2006; Tomko and Hochstrasser, 2011). Furthermore, Rpn12 has been shown to use a short C-terminal segment for binding the completed lid subcomplex, and this association is required for efficient incorporation of the lid into the regulatory particle (Tomko and Hochstrasser, 2011). Recent cryo-EM studies have located a helical bundle within the regulatory particle, and it has been suggested that this structure is composed of the lid subunits' C termini (Beck et al., 2012). However, the functional relevance and detailed topology of the bundle are unknown.

Here, we investigated the assembly mechanism of the proteasome lid. We show that the helices found at the $C$ terminus of each lid subunit form a peculiar bundle that governs an ordered self-assembly process. Moreover, we obtained an ab initio atomic model of this bundle based on our combinatorial search algorithm combined with previously reported crosslinking data. The resulting topology reveals how the helical bundle serves as an unusual structure that couples the completion of lid assembly with binding to the base and thus with the activation of Rpn11's deubiquitinase activity. Our data also suggest that this bundle represents an important architectural feature that likely enables the lid to tolerate conformational changes in the regulatory particle during substrate processing. In addition, these findings shed light on the mechanisms by which the related CSN may assemble.

\section{RESULTS}

The $\mathrm{C}$ terminus of each subunit in the lid is predicted to form one or more helices (Figure 1A). These C-terminal helices are highly conserved (Figure 1B) and have been suggested to form a helical bundle structure (Figure 1C; Beck et al., 2012). For several lid subunits, the structures of their individual MPN or PCl domains have been solved by X-ray crystallography, but in all cases their C-terminal helices were either truncated before crystallization or not resolved in the electron density (Boehringer et al., 2012; Pathare et al., 2012; Sanches et al., 2007) Based on the lack of ordered density and observed truncations during expression and purification, these C-terminal regions are likely flexible or flexibly attached to individual subunits when not incorporated in the lid.

To determine the function of the C-terminal helices as well as the architecture of the helical bundle, we utilized our recently developed heterologous $E$. coli expression system to produce yeast lid particles lacking C-terminal helices from individual subunits. These constructs contained $\mathrm{N}$-terminal fusions to three different subunits: a $\mathrm{His}_{6}$ tag on Rpn11, a FLAG epitope on Rpn7, and maltose binding protein (MBP) on Rpn6 (Figure 2A). This allowed us to assess lid assembly and observe subassemblies using affinity purification steps followed by gel filtration chromatography (Figures 2B and 2C).

\section{The C-Terminal Helices Are Essential for Lid Assembly}

First, we deleted the C-terminal helix of Rpn12 and found that only Rpn12 was absent from an otherwise complete lid complex (Figure 3A; Figure S1A available online). This is consistent with previous studies showing that Rpn12 requires its $C$ terminus for incorporation into the lid (Tomko and Hochstrasser, 2011). 
A
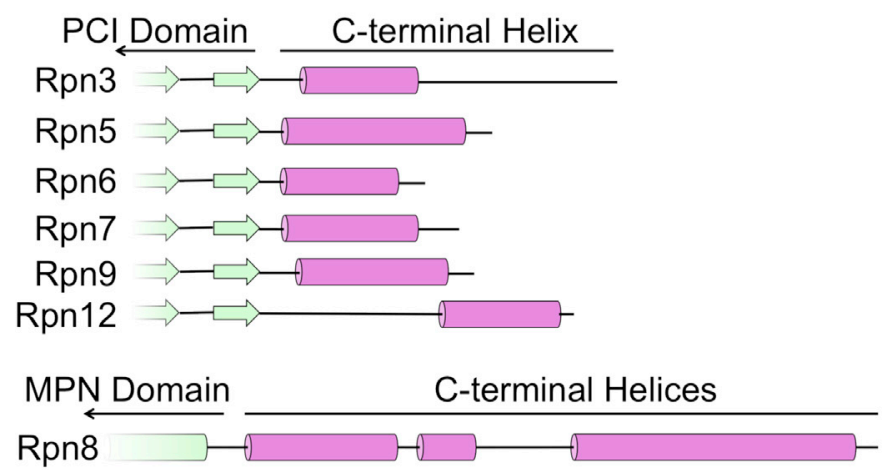

Rpn11

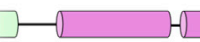

-1)
C

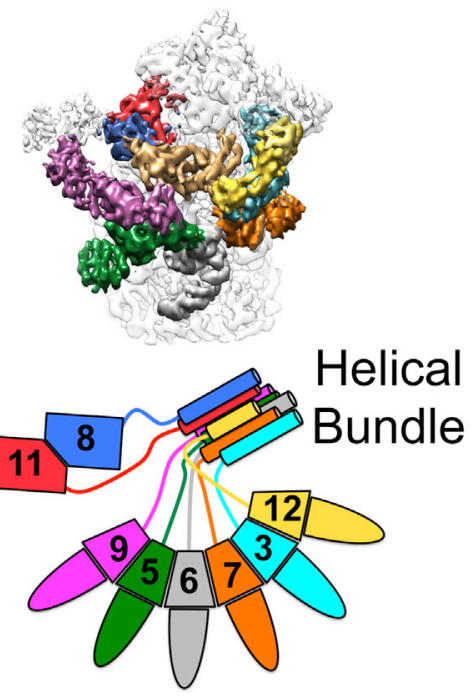

C-terminal Helix

B

Rpn7

PCI Domain

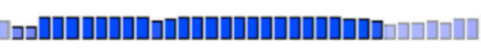

Confidence of secondary structure

Secondary structure prediction

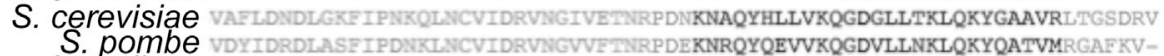

A. thalian VDYDRDLASETPDNKLNCVIDRVAGVVETNRP DEKNRQYQEVVKQGDVLLNKLQKYQATVMRGAFKV-

A. thallana VDEIDQELSRFIAAGKLHCKIDKVAGVLETMRPDAKNALYQATIKQGDFLLNRIQKLSRVIDL -

C. elegans RAFIDRELHRLIATGQLOCRIDAVIGVI EVNHRDSKNHLYKAVIKDGDILLNRIQKLARVINA------
D. melanogaster VEYIDQELARFIAAGRLHAKVDRVGGIVETNRPDNKNWQYQATIKQGDLLLNRIQKLSRVINI----

$H$ sapiens

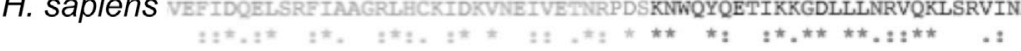

Figure 1. Conserved C-Terminal Helices of Individual Lid Subunits Form a Helical Bundle

(A) Secondary structure elements at the $C$ termini of individual lid subunits. $\beta$ sheets, random coils, and $\alpha$ helices are depicted as arrows, lines, and cylinders, respectively. MPN and PCI domain structures are in green and indicated above the secondary structure; the C-terminal helices are pink.

(B) The C-terminal helices are well conserved. As an example, a sequence alignment of Rpn7's C terminus is shown together with the secondary structure prediction and the prediction confidence.

(C) Proposed location of the lid helical bundle (beige) within the proteasome holoenzyme EM reconstruction (Beck et al., 2012). Lid subunits are individually colored and labeled as followed: Rpn3, cyan; Rpn5, green; Rpn6, gray; Rpn7, orange; Rpn8, blue; Rpn9, pink; Rpn11, red; Rpn12, gold. A similarly colored cartoon model of the lid is shown below the EM reconstruction.

Next, we produced particles lacking the C-terminal helix of Rpn5. Surprisingly, Rpn12 was missing from an otherwise completely assembled lid subcomplex (Figure 3B; Figure S1A). Similarly, we found that truncating the C-terminal helix of Rpn9 prevented Rpn12 from assembling with the lid complex (Figure 3C; Figure S1A). Together, these data suggest that the tails of Rpn5 and Rpn9, which previously had no presumed functions, are important for Rpn12 binding to the lid. Rpn12 had been suggested to use its $C$ terminus for monitoring a complete lid assembly state, but the mechanism of this surveillance remained elusive (Tomko and Hochstrasser, 2011). The individual tail truncations of Rpn5 and Rpn9 now suggest that the mechanism of surveillance by Rpn12 may be mediated through the C-terminal tails of the lid subunits.

Unlike most other lid subunits, Rpn3 contains a 45-amino-acid extension past its conserved C-terminal helix. This extra region is neither well conserved nor confidently predicted in its secondary structure. Upon deletion of the extension, we did not observe any lid assembly defects, suggesting that this portion of Rpn3 is not essential for complex formation (Figure S1B).
In contrast, deleting the conserved C-terminal helix of Rpn3 caused the lid to be separated into an Rpn5/6/8/9/11 subassembly, the Rpn3/7 heterodimer, and Rpn12, although for a small fraction the Rpn3/7 heterodimer was also associated with the Rpn5/6/8/9/11 assembly (Figure 3D; Figures S1B and S1C). Thus, Rpn3 and Rpn7 stably interact with each other through their $\mathrm{PCl}$ domains. We expect that the observed fractions of lid-bound and isolated Rpn3/7 heterodimer do not reflect their distribution under equilibrium conditions, given the extended purification procedure. Nevertheless, this partitioning indicates that the Rpn3/7 heterodimer has a reduced affinity for the Rpn5/6/8/9/11 subassembly when Rpn3's helix is missing.

We observed complementary results when Rpn7's C-terminal helix was deleted: the lid was separated into two subassemblies, Rpn3/7 and Rpn5/6/8/9/11, while Rpn12 did not associated with any members of the lid (Figure 3E; Figures S1B and S1C).

Deleting Rpn6's C-terminal helix led to the appearance of four components: Rpn5/8/9/11, Rpn3/7, Rpn6, and Rpn12 (Figure 3F; Figure S1D). Thus, Rpn6 relies on its own helix for lid binding, whereas Rpn3 and 7 depend not only on their own and each 


\section{Structure}

A

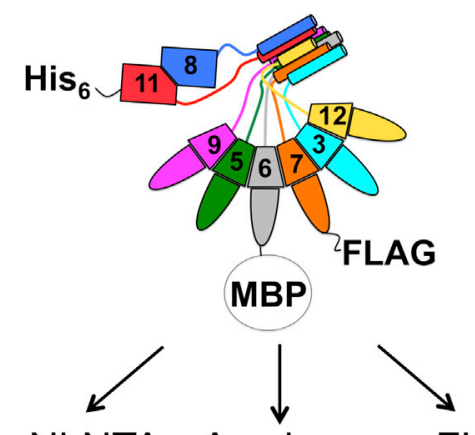

Ni-NTA Amylose $\alpha-$ FLAG

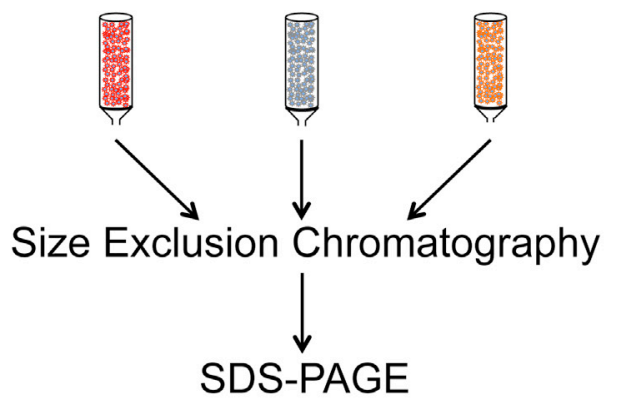

B

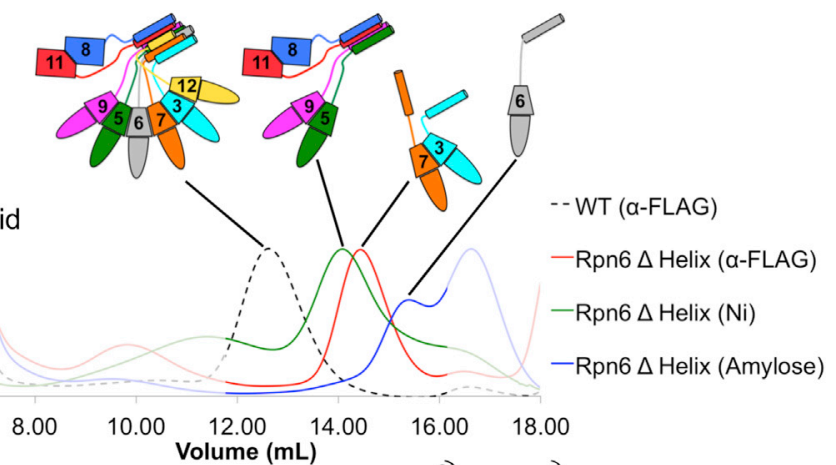

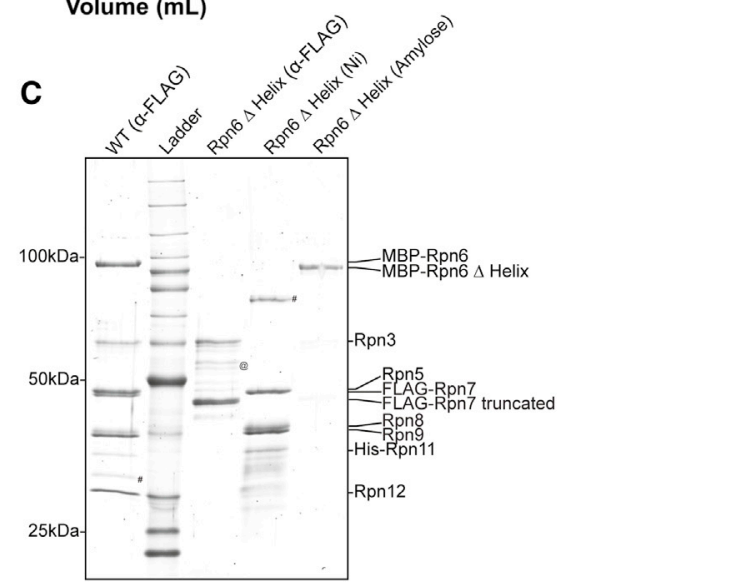

Figure 2. Experimental Design for Assessing Lid Assembly

(A) Schematic of the experimental setup. Individual subunits were pulled down from recombinant lid expressions using affinity chromatography (anti-FLAG, NiNTA, or amylose), and their assembly status was assessed with size-exclusion chromatography and SDS-PAGE. The schematic representation of the lid shows subunits individually colored as in Figure $1 \mathrm{C}$

(B) Representative size-exclusion chromatography traces after pulling down Rpn11, Rpn7, or Rpn6 from a recombinant lid expression including Rpn6 with a C-terminal helix deletion. Schematics above elution peaks indicate the compositions of subassemblies.

(C) Sypro-stained SDS gel showing the lid-subunit compositions present in the main elution peaks of the size-exclusion chromatography after pulling down Rpn11, Rpn7, or Rpn6 as described in (B).

other's helices, but also on that of Rpn6 for incorporation into the subcomplex. This is in agreement with previous pulldown experiments, showing that Rpn6 requires its C-terminal helix for interaction with Rpn7 (Pathare et al., 2012), and with experiments that indicated lid-assembly defects when Rpn6 was C-terminally extended (Isono et al., 2007). Notably, both Rpn3 and Rpn7 were truncated in the Rpn3/7 heterodimer, suggesting that their C-terminal helices were susceptible to proteolytic cleavage in E. coli or in lysate when not assimilated into the lid (Figure S1D).

To test the role of the MPN-domain-containing subunits for lid assembly, we deleted all three predicted helices at the $\mathrm{C}$ terminus of Rpn11. The truncation caused the majority of lid particles to be separated into Rpn5/6/8/9/11 and Rpn3/7, while the remainder of particles contained all subunits except for Rpn12 (Figure 3G; Figure S1E). We also wanted to assess whether the three predicted C-terminal helices of Rpn11 contribute differently to lid assembly, and therefore deleted only the last one. The phenotype for this variant resembled that for the deletion of all three helices, albeit with a larger fraction of particles only lacking Rpn12 (Figure S1E).

Finally, deleting the three predicted helices at the $\mathrm{C}$ terminus of Rpn8 caused the most severe assembly defect (Figure $3 \mathrm{H}$;
Figure S1F). Rpn11 and the truncated Rpn8 were found to be associated as a heterodimer. Similarly, Rpn3 and Rpn7 were bound to each other, whereas Rpn6 and Rpn12 were monomeric and not present in any subassemblies. With our experimental setup, we were unable to distinguish whether Rpn5 and Rpn9 are isolated or form a heterodimer.

Interestingly, during expression and purification of the lid variant with truncated Rpn8, the helices of Rpn 11 were partially cleaved off, likely due to their increased accessibility when not assembled with other helices of lid subunits. Rpn8 and Rpn11 thus interacted solely through their MPN domains. This heterodimer had only been predicted based on domain docking into cryo-EM maps of the proteasome and based on homodimers that were observed in crystal structures of related MPN domains (Beck et al., 2012; Sanches et al., 2007).

Secondary structure prediction software infers a random coil between the end of each $\mathrm{PCl}$ domain and the start of the corresponding C-terminal helix (Figure 1A). Many of these linkers between the helical bundle and the $\mathrm{PCI}$ domain of individual subunits contain at least one proline residue. We hypothesized that these prolines may rigidify the linkers to arrange the $\mathrm{PCI}$ domains and facilitate their association in a horseshoe-shaped structure 
A Rpn12 $\Delta$ Helix

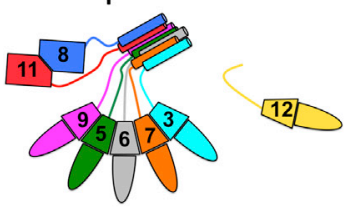

B Rpn5 $\Delta$ Helix

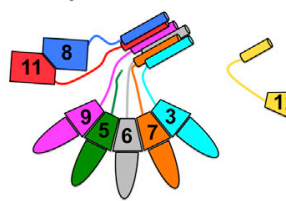

12

D Rpn3 $\Delta$ Helix
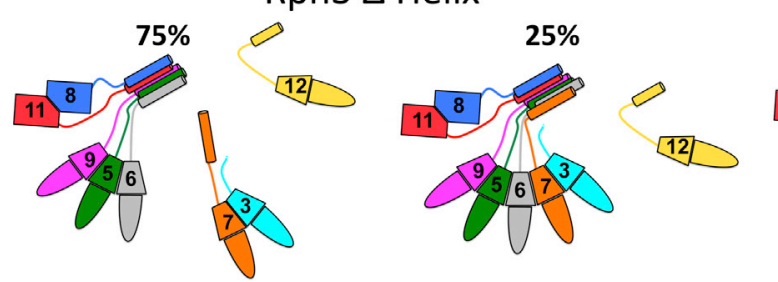

E Rpn7 $\Delta$ Helix
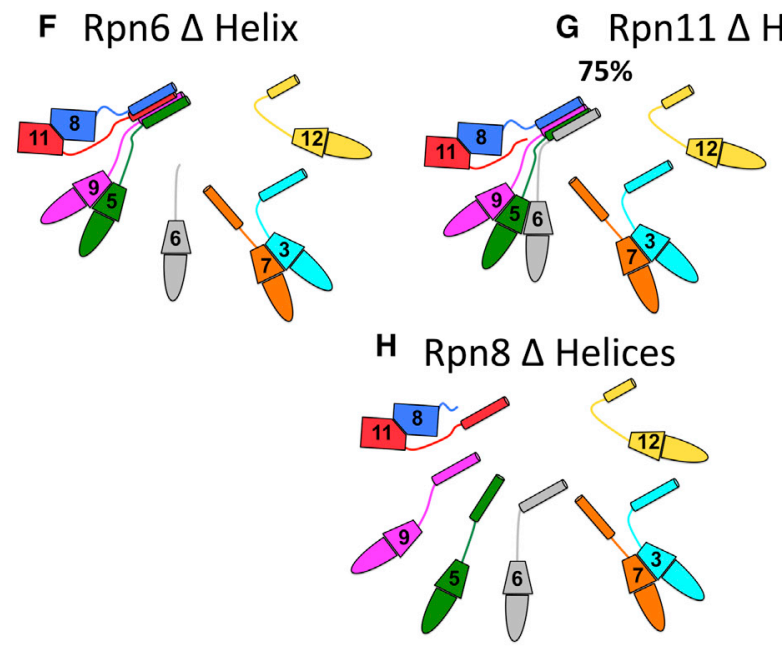

as the helical bundle assembles. To test this hypothesis, we mutated the linker regions of Rpn6 and Rpn7 from ETPN to ASAS and RPDN to ASAS, respectively, and analyzed the assembly phenotypes of these mutant lid constructs. In both cases, we observed complete assembly of the lid, suggesting that there is no sequence-specific role in assembly for the linkers (Figure S1G). These linkers of 5-20 residues may thus primarily function as flexible tethers between the helical bundle and the horseshoe-shaped structure of PCI domains.

\section{Lid Assembly Is Largely Independent of Individual PCI and MPN Domains}

Subnanometer cryo-EM structures indicated that six of the lid subunits form extensive contacts with lateral neighbors through their PCl domains. To determine the role of these interactions in lid assembly, we deleted the PCl domain of Rpn6, which is located in a central position within the horseshoe-shaped $\mathrm{PCl}$ arrangement. To our surprise, the presence of only the C-terminal helix of Rpn6 allowed a fraction of lid particles to fully assemble, with even Rpn12 attached (Figure 4A; Figure S1H). The other fraction was found in four pieces: Rpn3/7, Rpn5/8/9/ 11, Rpn12, and the C-terminal helix of Rpn6, which resembles the assembly defect observed when Rpn6's C-terminal helix is deleted. Based on these observations, we envision a scenario

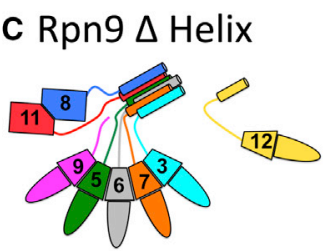

Figure 3. The C-Terminal Helices of the Lid Subunits Are Essential for Lid Assembly (A-H) Schematic representations of the lid subassemblies that were observed for the C-terminal helix truncations of individual subunits as indicated (see Figure S1 for the detailed analyses of subassemblies). Where noted, percentages reflect the relative abundance of different subassemblies. (H) Given our experimental setup and epitope placement, we are unable to distinguish whether Rpn5 and Rpn9 are separated (as shown) or form a heterodimer. wherein the absence of Rpn6's PCl domain causes the $\mathrm{PCl}$ hexamer to lose its rigid horseshoe shape, with the two remaining halves now connected only through the helical bundle, able to swivel independently of one another. Our data suggest that the $\mathrm{PCl}$ domain interactions contribute some of the binding energy between subunits, but lid assembly primarily relies on the bundle formation between their C-terminal helices.

To assess the role of the two MPN domains for lid assembly, we removed them simultaneously from Rpn8 and Rpn11. These domain deletions did not disrupt lid formation, demonstrating that the C-terminal helices of Rpn8 and Rpn11 are sufficient for complex assembly, whereas the MPN domains are dispensable for this process (Figure 4B; Figure $\mathrm{S} 1 \mathrm{H})$. This settles conflicting hypotheses in recent reviews suggesting that contacts between the MPN and PCI domains are important for lid assembly (Lander et al., 2013; Tomko and Hochstrasser, 2013).

Previous studies have shown that Rpn9 is not essential for cell viability in yeast. The lid purified from an Rpn9 deletion strain had been found to lack only Rpn 12 from an otherwise fully assembled subcomplex (Fukunaga et al., 2010). When we deleted the Rpn9 subunit in our recombinant system, the observed assembly phenotype paralleled the in vivo data, with lid lacking Rpn9 and only Rpn12 (Figure 4C; Figure S1H). Thus, our results support previous evidence that neither the C-terminal helix nor the $\mathrm{PCl}$ domain of Rpn9 is necessary for lid assembly with the exception of Rpn12.

Together, these data prove that the C-terminal helices of the lid subunits are essential and largely sufficient for lid self-assembly. Their association into the helical bundle as a well-defined three-dimensional structure provides most of the binding energy for complex formation. Furthermore, this bundle apparently forms a hub that allows Rpn12 to monitor the overall status of lid assembly. Our biochemical data suggest a number of likely contacts between helices within the bundle. For example, Rpn12 is sensitive to the absence of any other helix, suggesting that it directly interacts with several of them. Furthermore, the helix of Rpn6 is required for incorporation of the Rpn3/7 


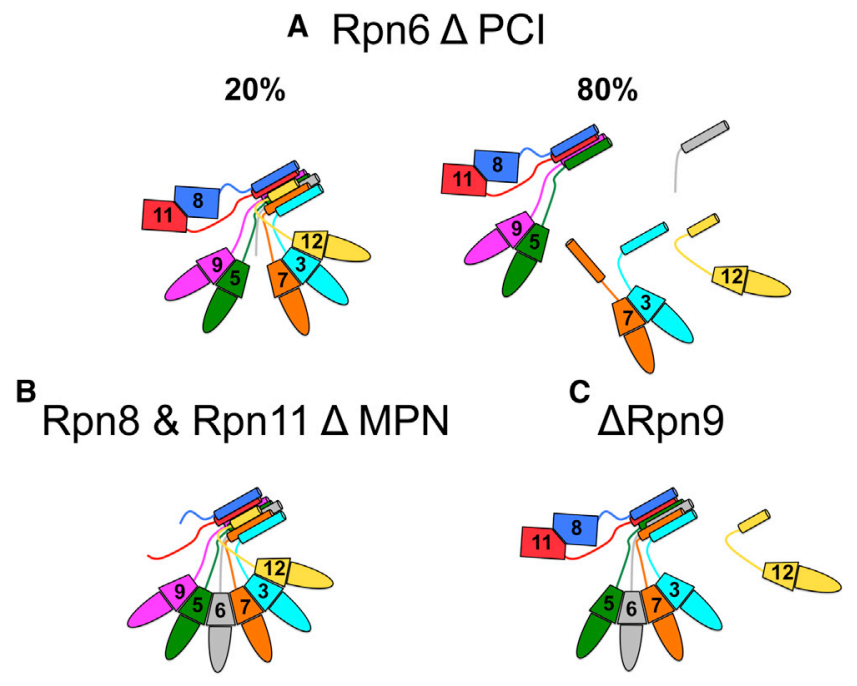

Figure 4. Role of MPN and PCI Domains for Lid Assembly (A) The lid is able to assemble even upon deletion of the PCl domain of Rpn6, which is located in the middle of the horseshoe-shaped $\mathrm{PCl}$ arrangement. Percentages reflect the relative abundance of two different assembly species for this Rpn6 PCl deletion.

(B) The MPN domains are not involved in lid assembly, as indicated by the phenotype observed for the deletion of MPN domains from Rpn8 and Rpn11. (C) The $\mathrm{PCl}$ domain of Rpn9 is neither required for assembly of the remaining $\mathrm{PCl}$ arrangement nor the association of the neighboring MPN-domaincontaining subunit Rpn8.

See also Figure S1.

heterodimer and therefore likely contacts one or both helices of this dimer. Based on the severe assembly phenotype observed upon C-terminal truncation of Rpn8, its helices may occupy a central position within the bundle. Given these biochemical results on bundle organization, we performed an independent determination of the bundle topology to provide an important structural framework.

Modeling Reveals the Topology of the Lid Helical Bundle Previous efforts to understand the bundle arrangement were only able to assign three helices within the 12-helix bundle, two from Rpn11 and one from Rpn8 (Beck et al., 2012). Determining which helix belongs to which protein is not trivial. For most subunits, the cryo-EM maps did not allow the tracing between helices and the respective MPN or $\mathrm{PCl}$ domains. In addition, the number of potential helix configurations in the bundle without constraints is enormous-for 12 helices, there are $12 ! \times 2^{12}\left(2 \times 10^{12}\right)$ possible nonredundant solutions.

To alleviate this massive computational problem, we developed a combinatorial search algorithm that recursively matches a set of geometric constraints (GCs) with a set of topology constraints (TCs; Figure 5). The TCs include the lengths of individual helices as well as the lengths of the linkers between the PCl domains and the bundle, as estimated from the secondary structure prediction of the lid subunits (Buchan et al., 2010; Table S1). The GCs were extracted from an $\sim 7 \AA$ resolution cryo-EM map (EMD: 2165; Beck et al., 2012), using a tracing algorithm to localize the individual helix densities within the bundle (Rusu et al., 2012; Table S2). Additional GCs were provided by the flex- ibly fitted structures of the PCl and MPN domains of all lid subunits (Protein Data Bank [PDB] code 4B4T; Beck et al., 2012). The last $\mathrm{C} \alpha$ atoms of these structures were taken as anchor points for the linkers connecting individual globular domains with the respective helices in the bundle (Table S2). Essentially, the problem is reduced to an iterative search of models for which predicted helix and linker lengths are compatible with the physical constraints observed in the cryo-EM map.

Intriguingly, with relatively few constraints and even using generous tolerances, our hybrid approach gave only four solutions (Table S3). These solutions shared the same assignment of helices, but differed in the orientations of the last helix of both Rpn8 and Rpn12. To determine the relative directions of these two helices, we further validated the four solutions against all 13 previously observed interlysine crosslinks that include at least one residue within the bundle (Table S4; Kao et al., 2012; Lasker et al., 2012). With these additional constraints, we were able to further narrow down the arrangement of helices to a single solution. The resulting topological model accommodated every reported crosslink, strongly supporting its validity. Furthermore, our helical bundle configuration is consistent with the previously reported placement of three helices from Rpn8 and Rpn11 (Beck et al., 2012), with the linker lengths required to connect a given $\mathrm{PCl}$ domain with the respective $\mathrm{C}$-terminal helix in the bundle (Table S5) and with the connecting electron densities observed for the linkers of Rpn5 and Rpn7 (Figure S2). Based on our predicted topology, a heavy-atom model of the helical bundle was generated and flexibly fitted into the experimental map with iMODFIT (Figures 6A and 6B). This model accounts for all density in the helical bundle region and also fits well into the other previously published subnanometer cryo-EM reconstruction of the yeast proteasome (Lander et al., 2012; Figure S3).

\section{The Bundle Architecture Explains Assembly Defects Observed for Helix Deletions}

The helical bundle is composed of twelve helices, with seven of them forming a structure reminiscent of a revolver cylinder (Figure 6C). In this cylinder, a central helix (the "center pin") is surrounded by six other helices (the "chambers"), which are slightly angled relative to the central one. The center pin position is occupied by the terminal helix of Rpn8, the longest predicted helix in the bundle. The C-terminal helices of Rpn12, Rpn3, Rpn7, Rpn6, and the final two helices of Rpn11 surround this terminal helix of Rpn8. The remaining five helices of the bundle pack against this seven-helix cylinder and each other (Figures 6A and 6B.). Importantly, this topology derived by our integrative approach is consistent with the biochemical data presented above.

In our recombinant system, truncation of Rpn8's helices results in a major assembly defect with only $\mathrm{Rpn} 8 / 11$ and Rpn3/7 still interacting through their MPN and PCl domains, respectively (Figure 3H). Thus, Rpn5 and Rpn9 rely on Rpn8's helices for assembly into the lid. The seven-helix cylinder structure is hollow when missing Rpn8's terminal helix, which serves as the lynchpin for assembly of each of the six surrounding helices (Figure 6C). Consistent with this model, we find that the C-terminal helix of Rpn8 exhibits high hydrophobicity over an $\sim 15$ amino acid segment, which is contacted by the six helices of the cylinder according to our derived bundle topology. 


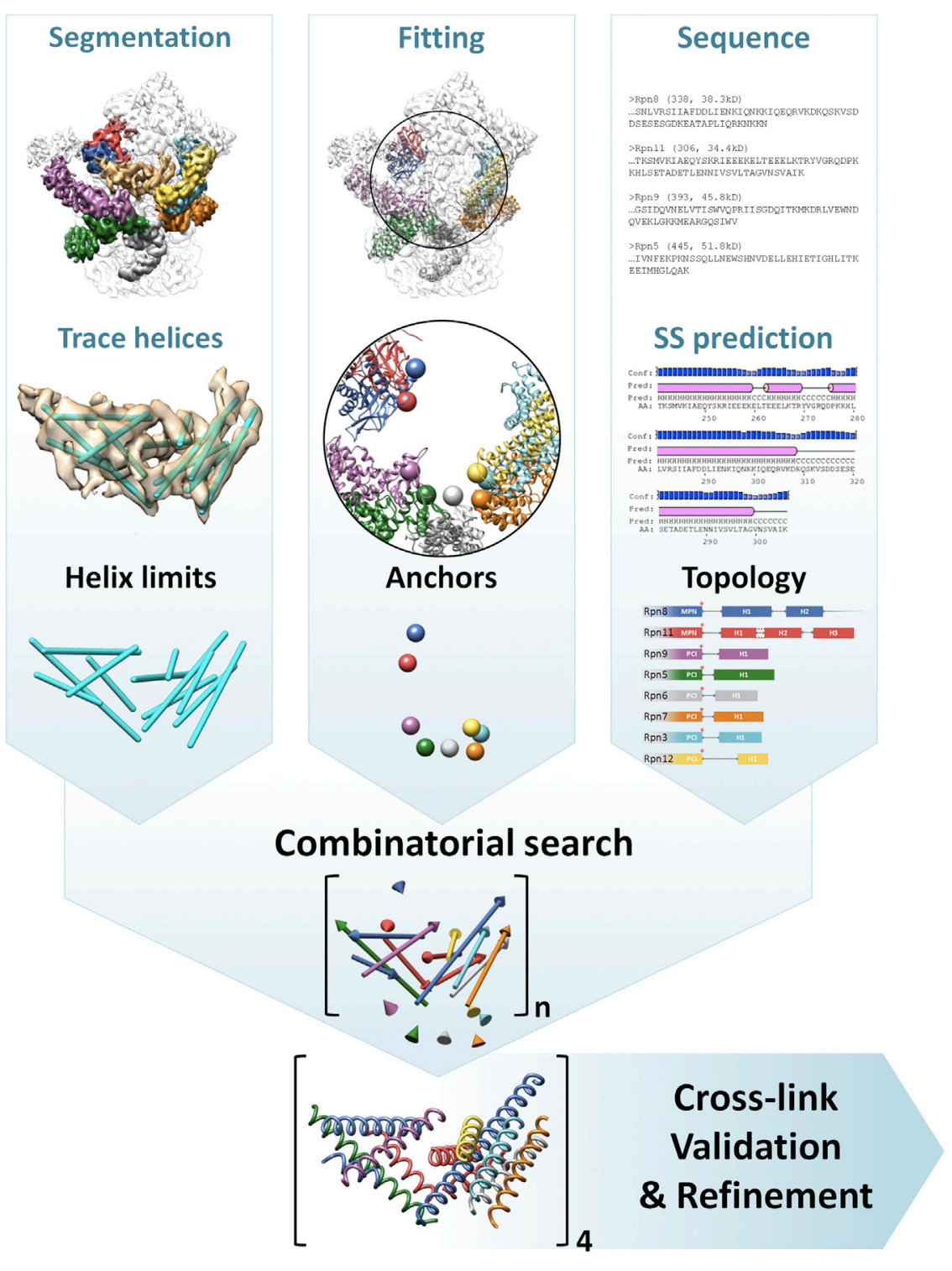

Figure 5. Modeling Workflow for Determining Helical Bundle Topology

The helical bundle region (yellow) was segmented from an $\sim 7 \AA$ resolution proteasome map (gray transparency, EMDB 2165; Beck et al., 2012), with lid subunits individually colored. Helix limits (cyan sticks) were automatically traced by the voltrac tool (Rusu and Wriggers, 2012) from the segmented map (yellow transparency).

The anchor points for the C-terminal helix linkers were extracted from the available fitted models of lid subunits (PDB code 4B4T; Beck et al., 2012) and are represented by colored spheres.

The topologies of individual S. cerevisiae lid subunits were predicted based on their sequences using the PSIPRED server (Buchan et al., 2010). Colored arrows represent one example of the helical bundle configurations tested during the combinatorial search. Note that the anchor points are now shown as cones pointing toward the connected helix.

Resulting models for the helical bundle with individual helices colored corresponding to the respective lid subunits. These models were further validated with existing crosslinking data (Kao et al., 2012; Lasker et al., 2012; Tomko and Hochstrasser, 2011) and finally refined by flexible fitting. See also Figure S2 and Tables S1-S4.

Based on our topological model, the terminal helix of Rpn11 contacts Rpn8 and Rpn9. However, its truncation caused only a minor assembly defect, indicating that the interactions with Rpn8 and Rpn9 are not integral for the quaternary structure of the bundle (Figure $3 G)$. Deletion of all three C-terminal helices of Rpn11 results in the formation of two lid species. One fraction of subunits is completely assembled, with the exception of Rpn12, while the other fraction assembles only partially into the Rpn5/6/8/9/11 and Rpn3/7 subcom-

The fact that the C-terminal helices of both Rpn3 and Rpn7 are required for stable incorporation of these subunits into the bundle is consistent with the observed positions of these helices, occupying two adjacent "chambers" of the cylinder structure (Figures 3D, 3E, and 6C). Although the helix of Rpn7 alone provides enough binding energy to allow a fraction of the Rpn3/7 heterodimer to associate with Rpn5/6/8/9/11, this incorporation is strengthened by the presence of the Rpn3 helix. Our structural model suggests that the interaction of Rpn3's helix with the final helix of Rpn8 provides this additional contact.

The absence of Rpn6's helix causes a similar assembly defect as observed for the truncation of Rpn3 or Rpn7, with the difference that Rpn6 is no longer present in complex with Rpn5/8/9/ 11. Our structural model is in agreement with this observation, because the helix of Rpn6 makes direct contact with the helix of Rpn7, which itself is necessary for incorporation of the Rpn3/7 heterodimer (Figure 3E). plexes (Figure $3 G$ ). Thus, the antepenultimate and penultimate helices of Rpn11 play a role but are not crucial for the interaction between Rpn8's terminal helix and Rpn3/7. Notably, this observation also implies that Rpn6 is able to bind to the lid in the absence of the other five 'chamber' helices (Rpn12, Rpn3, Rpn7, and both helices of Rpn11). The incorporation of Rpn6 is apparently supported by the $\mathrm{PCl}-\mathrm{PCl}$ interactions with its neighbor Rpn5, consistent with the destabilization observed for the lid complex upon deletion of Rpn6's PCl domain.

C-terminal truncations of Rpn5 or Rpn9 did not cause any major lid assembly defects but prevented the association of Rpn12 (Figures 3B and 3C). Based on our determined bundle topology, the helix of Rpn12 directly interacts with Rpn9 and thus is able to sense the presence of this subunit. However, there are no observed contacts between Rpn5 and Rpn12, suggesting that Rpn12 may detect the presence of Rpn5 indirectly through a repositioning of Rpn11's helices or through global conformational changes of the entire bundle induced by Rpn5. 


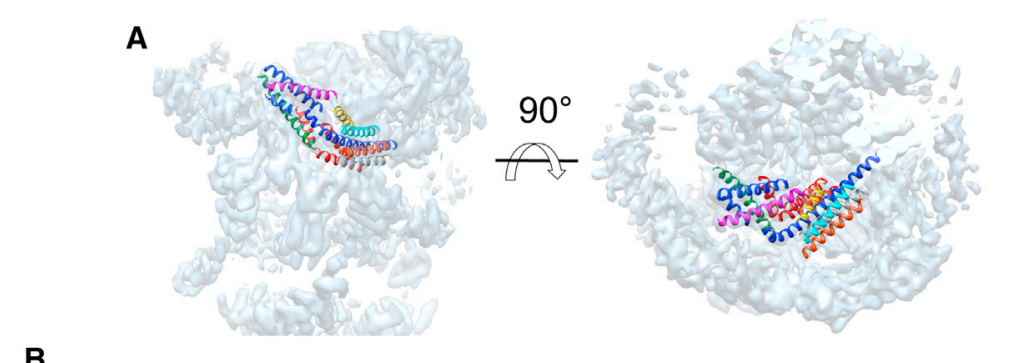

B
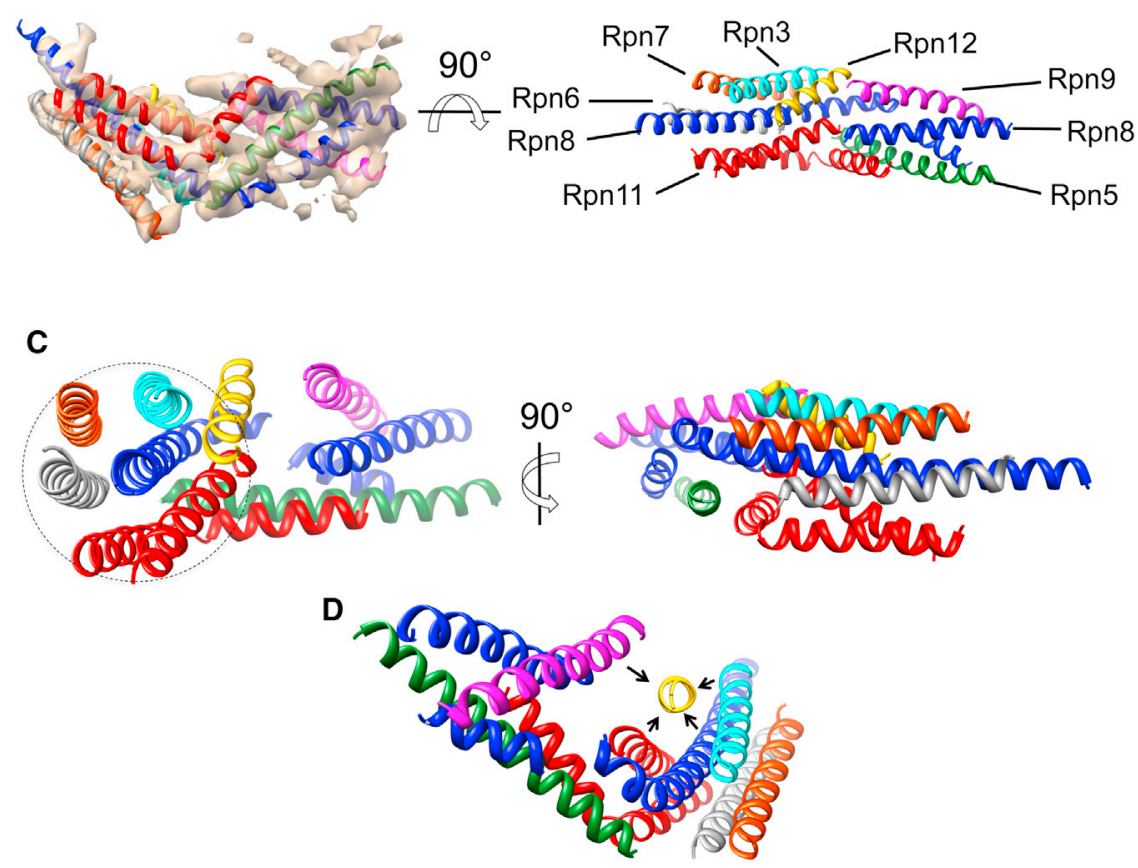

Rpn9 does not rely on a C-terminal helix for incorporation into the lid, but instead seems to depend on interactions between its $\mathrm{PCl}$ domain and the helical bundle or the neighboring $\mathrm{PCl}$ domain of Rpn5. Interestingly, Rpn5's association with other subunits is not only independent of its C-terminal helix, but also not affected by individually eliminating either of its $\mathrm{PCl}$ domain neighbors, Rpn6 and Rpn9. Additional interactions between the N-terminal portion of Rpn5 and the MPN domain of Rpn11 had been postulated based on our EM structure of the isolated lid (Lander et al., 2012). However, deletion of the Rpn8-Rpn11 MPN-domain dimer also did not disrupt Rpn5's association. Rpn5 thus appears to use several different interfaces to assemble with the lid.

The topology of the bundle suggests an elegant mechanism through which the incorporation of Rpn12's helix relies on the helices of every other subunit (Figure 6D). Although the 15-residue helix of Rpn12 contacts the helices of Rpn3, Rpn8, Rpn9, and Rpn11, these interactions likely include no more than two side chain contacts each. This relieves the dependence of the helical bundle assembly on the presence of Rpn12 because all helices that contact Rpn12 form many substantial interactions with other helices. In contrast, the helix of Rpn12 makes fewer contacts and thus seems to rely on the presence of all other lid subunits, either through direct interactions or indirectly through
Figure 6. Topological Organization of the Lid Helical Bundle

(A) Predicted heavy atom model for the helical bundle (PDB code 3J47) fitted into the regulatory particle EM density (Beck et al., 2012), with individual helices colored according to the respective subunit.

(B-D) Model for the helical bundle viewed from five different angles, with the left representation in (B) showing the fit into the EM density.

(C) The helices of Rpn3, Rpn6, Rpn7, Rpn8, Rpn11, and Rpn12 form a shape that resembles the cylinder of a revolver (indicated by a circle), with Rpn8 occupying the "center pin" position. The axes of the surrounding helices are slightly tilted relative to the central helix of Rpn8.

(D) The helix of Rpn12 directly contacts the helices of Rpn3, Rpn8, Rpn9, and Rpn11, as indicated by arrows.

See also Figure S3 and Table S5.

the overall bundle conformation. This feature is especially important considering that the $\mathrm{C}$ terminus of Rpn12 has previously been shown to be necessary for efficient association of the completed lid with the base subcomplex to form the regulatory particle (Tomko and Hochstrasser, 2011).

\section{DISCUSSION}

Here, we identified a helical bundle as a device that ensures the correct selfassembly of the proteasome lid subcomplex, provides the majority of the binding energy between eight lid subunits, and functions as a hub through which the lid monitors its own completion. The bundle is formed by the conserved C-terminal helices of the MPN- and PCl-domain-containing subunits, whose individual truncations revealed an ordered assembly mechanism. Furthermore, using hybrid methodology, we gained critical insights into the topology of the bundle, allowing us to interpret our biochemical data within a structural framework.

\section{Assembly Mechanism for the Lid}

Our findings reveal the striking ability of the large lid subcomplex to self-assemble as well as monitor its proper completion without the help of any specific assembly factors or chaperones. The assembly likely proceeds through an ordered addition of C-terminal helices into a bundle that thus allows a hierarchical complex construction (Figure 7). First, Rpn8 and Rpn11 dimerize through their MPN domains, whose helices then interact with the C-termini of Rpn5 and Rpn9. Next, Rpn6 binds through its C-terminal helix to the nascent Rpn5/8/9/11 assembly, followed by incorporation of the Rpn3/7 heterodimer. As the final assembly piece, Rpn12 binds through its $C$ terminus to the bundle, allowing the lid to incorporate into the regulatory particle. Although we used an E. coli-expression system that lacks 


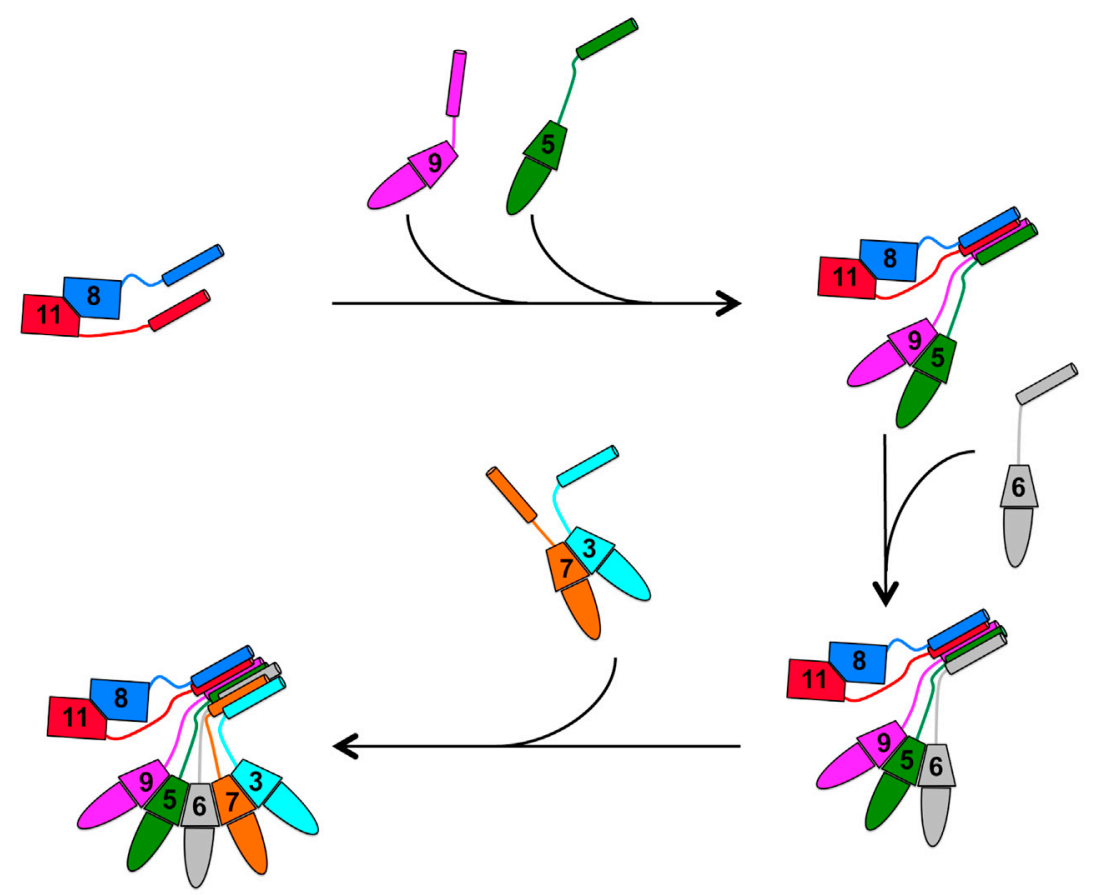

Figure 7. Model for Lid Assembly and Activation of Rpn11

Cartoon models depict the steps of lid assembly, which follows an ordered process guided by the C-terminal helices of individual subunits. Efficient association of the lid, the base, and the core to form the 26S proteasome holoenzyme requires complete lid assembly and activates the deubiquitinase of Rpn11 (indicated by red lines).

dicted by the SNARE's heptad repeat (Sutton et al., 1998). For the lid subcomplex, Rpn9, 11, and 12 possess helices predicted to form coiled coils, and future structural work at atomic resolution will be required to define the detailed interactions within the lid helical bundle.

\section{The Helical Bundle Acts as a}

Suspended Assembly Hub

The results presented here show that the helical bundle serves as a hub through which the $\sim 15$-residue C-terminal helix of Rpn12 can monitor the complete lid assembly (Figure 6D). Individual truncations revealed that Rpn12 is sensitive to the absence of any single helix within the eight-subunit bundle. The C-terminal location of all the helices may be another important design principle, ensuring that only fully translated subunits are incorporated into the lid.

Macromolecular assemblies often prevent their catalytic activity until complex maturation. Given the importance and diverse roles of ubiquitin signaling in the cell, it may be necessary to prevent the rogue deubiquitination of proteins by proteasome assembly intermediates outside the holoenzyme context. The deubiquitinase activity of Rpn11 is therefore inhibited until lid incorporation into the regulatory particle (Verma et al., 2002), presumably by allosteric interactions

posttranslational modifications or other potential factors, our derived mechanistic assembly model is consistent with previously reported endogenous subassemblies isolated from yeast (Fukunaga et al., 2010; Isono et al., 2007; Tomko and Hochstrasser, 2011). Our structural model predicts direct contacts between most of the C-terminal helices, which thus are able to detect each other directly. However, some of the helices appear to indirectly sense others through conformational changes of mutual interaction partners or the entire bundle during lid formation.

The lid assembly mechanism is reminiscent of the four-helix bundle formation harnessed by the SNARE complex to induce vesicle fusion (Hanson et al., 1997). In the case of the SNARE bundle, the helices pack as a four-helix coiled-coil, a state pre- with the helical bundle or the $\mathrm{N}$ terminus of Rpn5 (Beck et al., 2012; Lander et al., 2012). Because efficient lid incorporation depends on the presence of Rpn12, the activation of Rpn11 is elegantly linked to the complete assembly of the helical bundle (Figure 7).

The helical bundle likely has additional functional properties. For instance, it links the MPN domain dimer and the horseshoe-shaped arrangement of $\mathrm{PCl}$ domains through flexible tethers, allowing a certain degree of independent movements of these structural entities. This flexibility may be important for proteasome function because different portions of the regulatory particle have been observed to undergo significant differential movements during substrate engagement and degradation (Matyskiela et al., 2013). Moreover, the AAA+ ATPases of the 
base are expected to transition through substantial conformational changes during their cycles of ATP binding, hydrolysis, and nucleotide release, which may require a flexible attachment of the lid. In fact, two significant contacts are made between the bundle and the base. The C-terminal helices of Rpn3 and Rpn8 contact the coiled-coil of Rpt6, while the terminal helix of Rpn11 contacts the OB-fold of Rpt3. Additional studies will be required to define the functional implications of these contacts.

The helix interactions within the bundle appear to provide most of the energy for the assembly of lid subunits, whereas the weaker $\mathrm{PCl}-\mathrm{PCl}$ interactions may primarily contribute specificity and thus determine the subunit order within the horseshoe-shaped arrangement of $\mathrm{PCl}$ subunits. This specificity contribution might be important considering that highly homologous $\mathrm{PCl}$ domain subunits are also present in other macromolecular complexes such as elF3 and the CSN (Pick et al., 2009; Sun et al., 2011). The requirement of the bundle for lid assembly could thus prevent the incorporation of $\mathrm{PCl}$-containing subunits from other complexes. Furthermore, weaker lateral PCI-PCI interactions may provide flexibility within the horseshoe arrangement and allow certain conformational changes within the regulatory particle during substrate degradation (Matyskiela et al., 2013).

The helical bundle is also interesting from a protein folding perspective because its 12 helices are contributed by eight different polypeptides. To our knowledge, this is in contrast to all previously described helical bundles of similar size. The association of eight polypeptides into this structure likely imposes a large entropic cost, and how this burden is overcome may be revealed by future studies on the energetics of bundle formation. Additionally, the bundle may have potential for synthetic biology applications and the ordered assembly of designed circuits by attaching short C-terminal helices to protein components.

\section{Lid and CSN Likely Use Similar Assembly Strategies}

The CSN regulates the activity of the cullin-RING family of E3 ligases through removal of Nedd8, an ubiquitin-like moiety, from cullin subunits (Cope et al., 2002). Given the strong structural and topological homologies between the lid and the CSN, we predict that both complexes assemble by very similar mechanisms using a helical bundle. Like the lid, the CSN consists of MPN- and PCl-domain-containing subunits that, according to secondary structure predictions, all contain C-terminal helices. We thus anticipate that these helices play a vital role in CSN assembly. Interestingly, two recent reports document the in vivo and in vitro assembly of the CSN strictly requiring the C-terminal helices but not the MPN domain of Csn6 (the paralog of the lid Rpn8) (Kotiguda et al., 2012; Pick et al., 2012). Moreover, deletion of Csn5 (the paralog of the lid Rpn11) from a recombinant expression system for the human CSN allowed an otherwise complete assembly of the complex (Enchev et al., 2012). This result parallels our observation that neither the MPN domain nor the C-terminal helices of Rpn11 are necessary for lid assembly. Interestingly, though, while a truncation of Rpn11 prevents Rpn12 from associating with the lid, the equivalent truncation in the CSN still allows the incorporation of Csn8 (the Rpn12 paralog). This suggests that the CSN may not use its Rpn12 paralog to monitor proper complex formation. Finally, the $C$ terminus of Csn7 (paralog of the lid Rpn9) has been shown to be necessary and sufficient for binding to Csn6 (the paralog of Rpn8; Dessau et al., 2008), consistent with our results for the Rpn8/9 interactions. Similar to the lid, the CSN thus seems to utilize a helical bundle for the association of its subunits, and our lid assembly model may provide a valuable framework to explain and interpret previous results for this related complex.

In summary, our present study revealed several important features of the lid subcomplex that are critical for the assembly and function of the $26 \mathrm{~S}$ proteasome. The helical bundle drives lid formation and acts as a scaffold for monitoring the presence of every constituent within the subcomplex. It functions analogously to assembly factors by establishing an ordered assembly process and ensuring the proper arrangement of subunits. Completion of the bundle allows the lid to be incorporated into the holoenzyme, which has been shown previously to activate proteasomal deubiquitinase activity. Furthermore, we hypothesize that the helical bundle allows the lid to tolerate conformational changes in the regulatory particle during substrate processing. The helical bundle of the proteasome lid therefore exhibits a number of features that may be used by other macromolecular complexes, including the related $\mathrm{PCl}$-containing assemblies of CSN and elF3.

\section{EXPERIMENTAL PROCEDURES}

Recombinant Lid Construction and Purification

Plasmids for recombinant lid production were the same as described previously, with the exception of the addition of an N-terminal MBP (maltose binding protein) to Rpn6 (Lander et al., 2012). However, the Rpn8 and Rpn11 $\Delta$ MPN construct lacked the MBP tag on Rpn6. Truncations of individual subunits were produced using conventional cloning techniques. Lid proteins were expressed in E. coli BL21-star (DE3) as described previously (Lander et al., 2012). Cells were collected by centrifugation $(4,000 \times g$ for $30 \mathrm{~min})$, resuspended in FLAG buffer (50 mM HEPES, pH 7.6, $100 \mathrm{mM} \mathrm{NaCl}, 100 \mathrm{mM} \mathrm{KCl}$, and $5 \%$ glycerol) supplemented with protease inhibitors and $2 \mathrm{mg} / \mathrm{ml}$ lysozyme, and sonicated on ice for $2 \mathrm{~min}$ in $15 \mathrm{~s}$ bursts. The lysate was clarified by centrifugation $(27,000 \times g$ for $30 \mathrm{~min})$, and lid assemblies were affinitypurified either using anti-FLAG M2 resin (Sigma-Aldrich), amylose resin (New England Biosciences), or Ni-NTA agarose resin (QIAGEN), selecting for FLAG-Rpn7, MBP-Rpn6, and $\mathrm{His}_{6}$-Rpn11, respectively. The protein was concentrated in a 30,000 MWCO concentrator (Amicon) for further purification on a Superose 6 size-exclusion column (GE Healthcare) equilibrated in FLAG buffer plus $1 \mathrm{mM}$ DTT (Figure 2A).

\section{Hybrid Approach for Helical Bundle Assignment}

We developed a two-step integrative approach to unravel the structure of the proteasome helical bundle. In the first step, a combinatorial search is performed to assign the helix predictions of lid $C$ termini with the physical constraints observed in the cryo-EM map. In the second step, the compatible models found in the search are further screened with available crosslink information.

EM Map-Derived Geometrical Constraints

The helical bundle region was extracted from the experimental proteasome map (EMDB code 2165; Beck et al., 2012) using the Situs package (Rusu et al., 2012). Twelve helical segments were automatically detected using a stochastic template-based search method (Rusu and Wriggers, 2012; Table S2). The last residues of the flexibly fitted globular structures for Rpn3, Rpn5, Rpn6, Rpn7, Rpn8, Rpn9, Rpn11, and Rpn12 (PDB code 4B4T; Beck et al., 2012) were taken as starting points for the combinatorial search. These anchor points and the positions of the traced helices were used as geometric constraints (Table S2).

Topological Constraints

The secondary structure predictions have been carried out with PSIPRED (Buchan et al., 2010) from the corresponding lid C-terminal sequences (Table S1). The topological constraints (TC) were calculated from the predicted linker and helix lengths. The helix lengths were estimated by multiplying the 
corresponding number of predicted helix residues by $1.5 \AA$. We used a maximum inter-residue linker length of $2.9 \AA$ computed from a representative benchmark of $\sim 200$ loops (Chys and Chacon, 2013).

Combinatorial Search

The combinatorial search algorithm recursively matched all TCs and GCs while allowing a certain tolerance to account for inaccuracies in the modeling procedures. By default, a broad tolerance was used. In fact, the linker lengths were extended by $\sim 9 \AA$ (three residues) and the length mismatch between predicted helices and helices observed in the EM map was set to $\pm 30 \%$. Because the number of helices present in the map must be greater or equal to the number of predicted helices, one predicted helix was split in two. It was apparent that only one of the longest predicted helices, Rpn8 (50 residues) or Rpn11 (40 residues), could match into the largest helix of the map (38 residues). Valid configurations were only obtained by splitting Rpn11 into two helices of 19 residues each, separated by a linker of two residues. Splitting the long helix of Rpn8 was an unsuccessful strategy and no valid configurations were obtained. Even using generous tolerances and relatively few constraints, the search yielded only four solutions (Table S3). All solutions shared the same assignment but differed in the orientation of the last helix of both Rpn8 and Rpn12. In general, the predicted helices fit very well into the assigned densities (Figure 6B; Figure S3). The discrepancy between the number of residues for the predicted versus EM-extracted helices was typically less than four residues. Crosslink Evaluation

For further validation, the helix configurations found in the combinatorial search were first idealized by a linear arrangement of $1.5 \AA$ spaced $C_{\alpha}$ atoms and then centered into the corresponding assigned EM helix. The crosslinks available for the helical bundle region were taken from Kao et al., 2012 and Lasker et al., 2012 and detailed in Table S4. The crosslink distances of the models were measured between the $\mathrm{C}_{\alpha}$ atoms of lysine residues from either the linear helices of the bundle or the fitted lid subunits. We defined a crosslink violation distance of $34 \AA$. This crosslinking distance was calculated by adding $5 \AA$ to previously reported crosslink distances estimated with crystallographic structures (Chen et al., 2010; Seebacher et al., 2006) to account for modeling inaccuracies in tracing, prediction, and fitting procedures. All the computed crosslink distances of the four solutions found in the combinatorial search were compatible. However, incompatible distances of $41-42 \AA$ were found for solutions 1 and 2, which were therefore rejected. The remaining solutions 3 and 4 only differed in the orientation of Rpn12's helix. Previous crosslinking experiments have shown that the last residue of Rpn12 is in proximity to the $\mathrm{N}$-terminal coiled-coil domain of Rpt3 (Tomko and Hochstrasser, 2011), which is only in agreement with solution 3 .

\section{Modeling}

The final model was further refined and extended to heavy atom representation with Chimera (Goddard et al., 2007). Moreover, the helices were manually rotated around their main axis to roughly expose the hydrophilic face to the solvent. Finally, this model was flexibly fitted into the helical bundle segmented map by using iMODFIT with default parameters.

Availability

This hybrid approach including the combinatorial search algorithm, helix modeling, and check procedures has been implemented in the Perl programming language and is distributed freely upon request. Further details of the approach will be described elsewhere.

\section{ACCESSION NUMBERS}

The PDB accession code for the predicted topological model of the helical bundle reported in this paper is $3 \mathrm{~J} 47$.

\section{SUPPLEMENTAL INFORMATION}

Supplemental Information includes three figures and five tables and can be found with this article online at http://dx.doi.org/10.1016/j.str.2013.06.023.

\section{ACKNOWLEDGMENTS}

We thank the members of the Martin lab and Susan Marqusee for helpful comments. This research was funded by NSF grant NSF-MCB-1150288 (to A.M.), the Searle Scholars Program (to A.M.), as well as grants CAM-S2010/BMD2353, HFSP-RGP0039/2008, and BFU2009-09552 (to P.C.).

E.E. designed, expressed, and purified proteasome lid constructs and performed and analyzed biochemical experiments; J.R.L.-B. and P.C. designed and developed the hybrid computational approach and modeled the helix bundle; all authors contributed to data analyses and manuscript preparation.

Received: May 20, 2013

Revised: June 15, 2013

Accepted: June 17, 2013

Published: August 1, 2013

\section{REFERENCES}

Arendt, C.S., and Hochstrasser, M. (1999). Eukaryotic 20 S proteasome cataIytic subunit propeptides prevent active site inactivation by $\mathrm{N}$-terminal acetylation and promote particle assembly. EMBO J. 18, 3575-3585.

Beck, F., Unverdorben, P., Bohn, S., Schweitzer, A., Pfeifer, G., Sakata, E., Nickell, S., Plitzko, J.M., Villa, E., Baumeister, W., and Förster, F. (2012). Near-atomic resolution structural model of the yeast $26 S$ proteasome. Proc. Natl. Acad. Sci. USA 109, 14870-14875.

Boehringer, J., Riedinger, C., Paraskevopoulos, K., Johnson, E.O., Lowe, E.D., Khoudian, C., Smith, D., Noble, M.E., Gordon, C., and Endicott, J.A. (2012). Structural and functional characterization of Rpn12 identifies residues required for Rpn10 proteasome incorporation. Biochem. J. 448, 55-65.

Buchan, D.W., Ward, S.M., Lobley, A.E., Nugent, T.C., Bryson, K., and Jones, D.T. (2010). Protein annotation and modelling servers at University College London. Nucleic Acids Res. 38(Web Server issue), W563-W568.

Chen, P., and Hochstrasser, M. (1996). Autocatalytic subunit processing couples active site formation in the $20 \mathrm{~S}$ proteasome to completion of assembly. Cell 86, 961-972.

Chen, Z.A., Jawhari, A., Fischer, L., Buchen, C., Tahir, S., Kamenski, T., Rasmussen, M., Lariviere, L., Bukowski-Wills, J.C., Nilges, M., et al. (2010). Architecture of the RNA polymerase II-TFIIF complex revealed by cross-linking and mass spectrometry. EMBO J. 29, 717-726.

Chys, P., and Chacon, P. (2013). Random Coordinate Descent with Spinormatices and Geometric Filters for Efficient Loop Closure. J. Chem. Theory Comput. 9, 1821-1829.

Cope, G.A., Suh, G.S., Aravind, L., Schwarz, S.E., Zipursky, S.L., Koonin, E.V., and Deshaies, R.J. (2002). Role of predicted metalloprotease motif of Jab1/ Csn5 in cleavage of Nedd8 from Cul1. Science 298, 608-611.

da Fonseca, P.C., He, J., and Morris, E.P. (2012). Molecular model of the human $26 \mathrm{~S}$ proteasome. Mol. Cell 46, 54-66.

Dessau, M., Halimi, Y., Erez, T., Chomsky-Hecht, O., Chamovitz, D.A., and Hirsch, J.A. (2008). The Arabidopsis COP9 signalosome subunit 7 is a model $\mathrm{PCl}$ domain protein with subdomains involved in COP9 signalosome assembly. Plant Cell 20, 2815-2834.

Enchev, R.I., Scott, D.C., da Fonseca, P.C., Schreiber, A., Monda, J.K., Schulman, B.A., Peter, M., and Morris, E.P. (2012). Structural basis for a reciprocal regulation between SCF and CSN. Cell Rep 2, 616-627.

Fukunaga, K., Kudo, T., Toh-e, A., Tanaka, K., and Saeki, Y. (2010). Dissection of the assembly pathway of the proteasome lid in Saccharomyces cerevisiae. Biochem. Biophys. Res. Commun. 396, 1048-1053.

Funakoshi, M., Tomko, R.J., Jr., Kobayashi, H., and Hochstrasser, M. (2009). Multiple assembly chaperones govern biogenesis of the proteasome regulatory particle base. Cell 137, 887-899.

Glickman, M.H., and Ciechanover, A. (2002). The ubiquitin-proteasome proteolytic pathway: destruction for the sake of construction. Physiol. Rev. 82, 373-428.

Glickman, M.H., Rubin, D.M., Coux, O., Wefes, I., Pfeifer, G., Cjeka, Z., Baumeister, W., Fried, V.A., and Finley, D. (1998). A subcomplex of the proteasome regulatory particle required for ubiquitin-conjugate degradation and related to the COP9-signalosome and elF3. Cell 94, 615-623.

Goddard, T.D., Huang, C.C., and Ferrin, T.E. (2007). Visualizing density maps with UCSF Chimera. J. Struct. Biol. 157, 281-287. 
Groll, M., Bajorek, M., Köhler, A., Moroder, L., Rubin, D.M., Huber, R., Glickman, M.H., and Finley, D. (2000). A gated channel into the proteasome core particle. Nat. Struct. Biol. 7, 1062-1067.

Hanson, P.I., Roth, R., Morisaki, H., Jahn, R., and Heuser, J.E. (1997). Structure and conformational changes in NSF and its membrane receptor complexes visualized by quick-freeze/deep-etch electron microscopy. Cell 90, 523-535.

Hughes, A.L. (1997). Evolution of the proteasome components. Immunogenetics 46, 82-92.

Isono, E., Nishihara, K., Saeki, Y., Yashiroda, H., Kamata, N., Ge, L., Ueda, T., Kikuchi, Y., Tanaka, K., Nakano, A., and Toh-e, A. (2007). The assembly pathway of the $19 \mathrm{~S}$ regulatory particle of the yeast $26 \mathrm{~S}$ proteasome. Mol. Biol. Cell 18, 569-580.

Kao, A., Randall, A., Yang, Y., Patel, V.R., Kandur, W., Guan, S., Rychnovsky, S.D., Baldi, P., and Huang, L. (2012). Mapping the structural topology of the yeast 19S proteasomal regulatory particle using chemical cross-linking and probabilistic modeling. Mol. Cell. Proteomics 11, 1566-1577.

Kotiguda, G.G., Weinberg, D., Dessau, M., Salvi, C., Serino, G., Chamovitz, D.A., and Hirsch, J.A. (2012). The organization of a CSN5-containing subcomplex of the COP9 signalosome. J. Biol. Chem. 287, 42031-42041.

Kusmierczyk, A.R., Kunjappu, M.J., Funakoshi, M., and Hochstrasser, M. (2008). A multimeric assembly factor controls the formation of alternative $20 S$ proteasomes. Nat. Struct. Mol. Biol. 15, 237-244.

Lander, G.C., Estrin, E., Matyskiela, M.E., Bashore, C., Nogales, E., and Martin, A. (2012). Complete subunit architecture of the proteasome regulatory particle. Nature 482, 186-191.

Lander, G.C., Martin, A., and Nogales, E. (2013). The proteasome under the microscope: the regulatory particle in focus. Curr. Opin. Struct. Biol. 23, 243-251.

Lasker, K., Förster, F., Bohn, S., Walzthoeni, T., Villa, E., Unverdorben, P., Beck, F., Aebersold, R., Sali, A., and Baumeister, W. (2012). Molecular architecture of the $26 \mathrm{~S}$ proteasome holocomplex determined by an integrative approach. Proc. Natl. Acad. Sci. USA 109, 1380-1387.

Matyskiela, M.E., Lander, G.C., and Martin, A. (2013). Conformational switching of the 26S proteasome enables substrate degradation. Nat. Struct. Mol. Biol. 20, 781-788.

Murata, S., Yashiroda, H., and Tanaka, K. (2009). Molecular mechanisms of proteasome assembly. Nat. Rev. Mol. Cell Biol. 10, 104-115.

Pathare, G.R., Nagy, I., Bohn, S., Unverdorben, P., Hubert, A., Körner, R., Nickell, S., Lasker, K., Sali, A., Tamura, T., et al. (2012). The proteasomal subunit Rpn6 is a molecular clamp holding the core and regulatory subcomplexes together. Proc. Natl. Acad. Sci. USA 109, 149-154.

Pereira-Leal, J.B., Levy, E.D., Kamp, C., and Teichmann, S.A. (2007). Evolution of protein complexes by duplication of homomeric interactions. Genome Biol. 8, R51.

Pick, E., Hofmann, K., and Glickman, M.H. (2009). PCl complexes: Beyond the proteasome, CSN, and elF3 Troika. Mol. Cell 35, 260-264.

Pick, E., Golan, A., Zimbler, J.Z., Guo, L., Sharaby, Y., Tsuge, T., Hofmann, K., and Wei, N. (2012). The minimal deneddylase core of the COP9 signalosome excludes the Csn6 MPN- domain. PLoS ONE 7, e43980.
Rusu, M., and Wriggers, W. (2012). Evolutionary bidirectional expansion for the tracing of alpha helices in cryo-electron microscopy reconstructions. J. Struct. Biol. 177, 410-419.

Rusu, M., Starosolski, Z., Wahle, M., Rigort, A., and Wriggers, W. (2012). Automated tracing of filaments in $3 \mathrm{D}$ electron tomography reconstructions using Sculptor and Situs. J. Struct. Biol. 178, 121-128.

Sanches, M., Alves, B.S., Zanchin, N.I., and Guimarães, B.G. (2007). The crystal structure of the human Mov34 MPN domain reveals a metal-free dimer. J. Mol. Biol. 370, 846-855.

Sauer, R.T., and Baker, T.A. (2011). AAA+ proteases: ATP-fueled machines of protein destruction. Annu. Rev. Biochem. 80, 587-612.

Scheel, H., and Hofmann, K. (2005). Prediction of a common structural scaffold for proteasome lid, COP9-signalosome and elF3 complexes. BMC Bioinformatics 6, 71.

Seebacher, J., Mallick, P., Zhang, N., Eddes, J.S., Aebersold, R., and Gelb, M.H. (2006). Protein cross-linking analysis using mass spectrometry, isotope-coded cross-linkers, and integrated computational data processing. J. Proteome Res. 5, 2270-2282.

Serino, G., and Pick, E. (2013). Duplication and familial promiscuity within the proteasome lid and COP9 signalosome kin complexes. Plant Sci. 203-204, 89-97.

Sharon, M., Taverner, T., Ambroggio, X.I., Deshaies, R.J., and Robinson, C.V. (2006). Structural organization of the 19S proteasome lid: insights from MS of intact complexes. PLoS Biol. 4, e267.

Smith, D.M., Chang, S.C., Park, S., Finley, D., Cheng, Y., and Goldberg, A.L. (2007). Docking of the proteasomal ATPases' carboxyl termini in the 20S proteasome's alpha ring opens the gate for substrate entry. Mol. Cell 27, 731-744.

Staley, J.P., and Woolford, J.L., Jr. (2009). Assembly of ribosomes and spliceosomes: complex ribonucleoprotein machines. Curr. Opin. Cell Biol. $21,109-118$

Sun, C., Todorovic, A., Querol-Audí, J., Bai, Y., Villa, N., Snyder, M., Ashchyan, J., Lewis, C.S., Hartland, A., Gradia, S., et al. (2011). Functional reconstitution of human eukaryotic translation initiation factor 3 (elF3). Proc. Natl. Acad. Sci. USA 108, 20473-20478.

Sutton, R.B., Fasshauer, D., Jahn, R., and Brunger, A.T. (1998). Crystal structure of a SNARE complex involved in synaptic exocytosis at $2.4 \mathrm{~A}$ resolution. Nature 395, 347-353.

Thrower, J.S., Hoffman, L., Rechsteiner, M., and Pickart, C.M. (2000). Recognition of the polyubiquitin proteolytic signal. EMBO J. 19, 94-102.

Tomko, R.J., Jr., and Hochstrasser, M. (2011). Incorporation of the Rpn12 subunit couples completion of proteasome regulatory particle lid assembly to lid-base joining. Mol. Cell 44, 907-917.

Tomko, R.J., Jr., and Hochstrasser, M. (2013). Molecular architecture and assembly of the eukaryotic proteasome. Annu. Rev. Biochem. 82, 415-445.

Verma, R., Aravind, L., Oania, R., McDonald, W.H., Yates, J.R., 3rd, Koonin, E.V., and Deshaies, R.J. (2002). Role of Rpn11 metalloprotease in deubiquitination and degradation by the $26 \mathrm{~S}$ proteasome. Science $298,611-615$. 